\title{
GEOQUÍMICA DE CAMADAS VERMELHAS BIOTURBADAS DA FORMAÇÃO AL- TER DO CHÃO, CRETÁCEO DA BACIA DO AMAZONAS
}

\author{
ADRIANA MARIA COIMBRA HORBE ${ }^{1}$, LUCIETH CRUZ VIEIRA ${ }^{1} \&$ \\ AFONSO CÉSAR RODRIGUES NOGUEIRA ${ }^{1}$
}

\begin{abstract}
Resumo Camadas de arenitos e pelitos vermelhos bioturbados são feições comumente encontradas na sucessão aflorante cretácea da Formação Alter do Chão, região NE do Estado do Amazonas. A análise geoquímica destas camadas em combinação com dados sedimentológicos e mineralógicos forneceram parâmetros para auxiliar no entendimento dos processos formadores e das condições climáticas e paleoambientais. Duas associações de elementos maiores e traços foram identificadas $\mathrm{K} O \mathrm{O}-\mathrm{Ba}-\mathrm{Mo}-\mathrm{Ni}$ - $\mathrm{Sr}-\mathrm{Y}$ e $\mathrm{Fe}_{2} \mathrm{O}_{3}-\mathrm{TiO}_{2}-\mathrm{Cd}-\mathrm{Cr}-\mathrm{V}-\mathrm{Zn}-\mathrm{Zr} / \mathrm{SiO}_{2}$ que combinadas refletiram três assinaturas geoquímicas principais: 1- elevados teores em $\mathrm{SiO}_{2}$ e baixos conteúdos em elementos-traço; 2- maior conteúdo de $\mathrm{K}_{2} \mathrm{O}, \mathrm{Ba}, \mathrm{Mo}, \mathrm{Ni}$, Sr e Y; e 3- ampla variação química, mas intermediária entre as assinaturas 1 e 2. Essas assinaturas marcam os depósitos de planícies abandonadas e rios entrelaçados da Formação Alter do Chão formados sob condições de clima seco com tendência a úmido, e sugerem variação de rochas fonte, seleção granulométrica durante o transporte e deposição, variações do grau de porosidade e pedogênese pós-deposicional.
\end{abstract}

Palavras-chave: Bacia do Amazonas, Cretáceo, Formação Alter do Chão

\begin{abstract}
GEOCHEMISTRY OF BIOTURBATED RED BEDS FROM ALTER DO CHÃO FORMATION, CRETACEOUS OF AMAZON BASIN. Reddish bioturbated sandstones and pelites beds are significant features of outcropping succession of Alter do Chão Formation, northeastern Amazonas State. Geochemistry analysis of reddish beds combine with mineralogical and sedimentological data provided parameters for better understanding the generating processes, climatic influence and paleoenvironmental conditions. Two associations of major and trace elements were identified $\mathrm{K}_{2} \mathrm{O}-\mathrm{Ba}-\mathrm{Mo}-\mathrm{Ni}-\mathrm{Sr}-\mathrm{Y}$ and $\mathrm{Fe}_{2} \mathrm{O}_{3}-\mathrm{TiO}_{2}-\mathrm{Cd}-\mathrm{Cr}$ $-\mathrm{V}-\mathrm{Zn}-\mathrm{Zr} / \mathrm{SiO}_{2}$ which providing three main geochemistry signatures: 1- high $\mathrm{SiO}_{2}$ and low trace elements contents; 2- high $\mathrm{K}_{2} \mathrm{O}, \mathrm{Ba}, \mathrm{Mo}, \mathrm{Ni}, \mathrm{Sr}$ e $\mathrm{Y}$ contents and 3- large chemical variation yet intermediary between 1 and 2 signatures. These signatures are closely related with abandoned floodplains and braided river channels of Alter do Chão Formation under dry to wet climatic conditions, and suggest source-area changes, granulometric sorting during transport and deposition, porosity degree modifications and post-depositional pedogenesis.
\end{abstract}

Keywords: Amazon Basin, Late Cretaceous, Alter do Chão Formation.

INTRODUÇÃO Camadas de arenitos e pelitos com intensa coloração vermelha são feições conspícuas dos depósitos cretáceos da Formação Alter do Chão da Bacia do Amazonas. Estas camadas apresentam diversas estruturas de origem biogênica (icnofósseis) e pedogenéticas (peds, slickensides) e podem ser friáveis ou silicificadas e, neste caso, são interpretadas como silcretes (Nogueira et al. 1999). Outros níveis bioturbados vermelhos não silicificados ainda não foram adequadamente interpretados na sucessão e não há detalhamento geoquímico e mineralógico para contribuir na definição das condições ambientais da época de sua formação que possam indicar evidências de superimposição de processos diagenéticos e supergênicos recentes.

A Formação Alter do Chão é a unidade de maior extensão aflorante na Bacia do Amazonas e está limitada pelos arcos de Purus a oeste e de Gurupá a leste, e é interpretada como produto de um sistema deposicional flúvio-deltáico-lacustre com planícies abandonadas de rios entrelaçados (Kistler 1954, Wanderley Filho 1994, Caputo et al. 1972, Souza 1974, Pereira 1988, Cunha et al. 1994, Viera \& Nogueira 1998, Dino et al. 1999, Nogueira et al. 2003). Além dos arenitos e pelitos com intensa coloração vermelha, é composta de rochas siliciclásticas avermelhadas que incluem arenitos feldspáticos caulínicos, quartzo-arenitos e quartzo-grauvacas com estratificação cruzada e intercalações de argilitos, siltitos e conglomerados, bem como brechas intraformacionais (Derby 1879, Souza \& Medeiros 1972, Cunha et al. 1994). Níveis silicificados, nesta unidade, são designados informalmente como Arenito Manaus (Albuquerque 1922). A idade é considerada como Cretácea Superior (Alagoas Superior a Cenomaniano).

$\mathrm{O}$ estudo das camadas de arenitos e pelitos com intensa co- loração vermelha dos depósitos aflorantes da Formação Alter do Chão na região de Manaus e Presidente Figueiredo é o tema deste trabalho. A integração de dados sedimentológicos, mineralógicos e geoquímicos tem por objetivo definir parâmetros geoquímicos para auxiliar o entendimento paleoambiental e a correlação dos processos formadores destas camadas vermelhas.

MATERIAIS E MÉTODOS Foram coletadas amostras de cinco afloramentos com camadas de cor vermelha intensa nas localidades de Ponta das Pedras (PP), Ponta Negra (PN), Ponta das Lajes (PL) e nos quilômetros 10 e 110 da rodovia BR 174 (Fig. 1). A composição mineralógica foi obtida por difração de raios-x e MEV e as amostras para análises químicas dos elementos maiores foram abertas por fusão com meta e tetraborato de lítio com posterior dissolução ácida (ácido clorídrico, perclórico e fluorídrico). Nestas foram quantificados silício por gravimetria, alumínio por titulometria e ferro total por espectrofotometria. A perda ao fogo foi analisada por gravimetria sem necessidade de abertura. Uma segunda etapa de abertura ácida foi utilizada para analisar $\mathrm{CaO}, \mathrm{MgO} \mathrm{Na} \mathrm{O}_{2}$ e $\mathrm{K}_{2} \mathrm{O}$ por absorção atômica, $\mathrm{As}$ e $\mathrm{Sb}$ com geração de hidretos, $\mathrm{Hg}$ por vapor frio e $\mathrm{Ag}, \mathrm{B}, \mathrm{Ba}, \mathrm{Be}, \mathrm{Bi}$, $\mathrm{Cd}, \mathrm{Co}, \mathrm{Cr}, \mathrm{Cu}, \mathrm{Li}, \mathrm{Mn}, \mathrm{Mo}, \mathrm{Ni}, \mathrm{Pb}, \mathrm{Sc}, \mathrm{Sn}, \mathrm{Sr}, \mathrm{V}, \mathrm{W}, \mathrm{Y}, \mathrm{Zn}, \mathrm{Zr}$ e ETR por espectrometria óptica de plasma de indução acoplada. Os dados das análises mineralógicas e químicas foram processados por estatística multivariada, com o programa Statistica 5.1. Foram identificados os elementos mais relevantes na caracterização geoquímica dos níveis estudados e o grau de correlação entre as amostras com base na análise fatorial. Para tal, foram selecionados todos os dados químicos exceto os ETR, pois estes foram analisados somente em parte das amostras. 


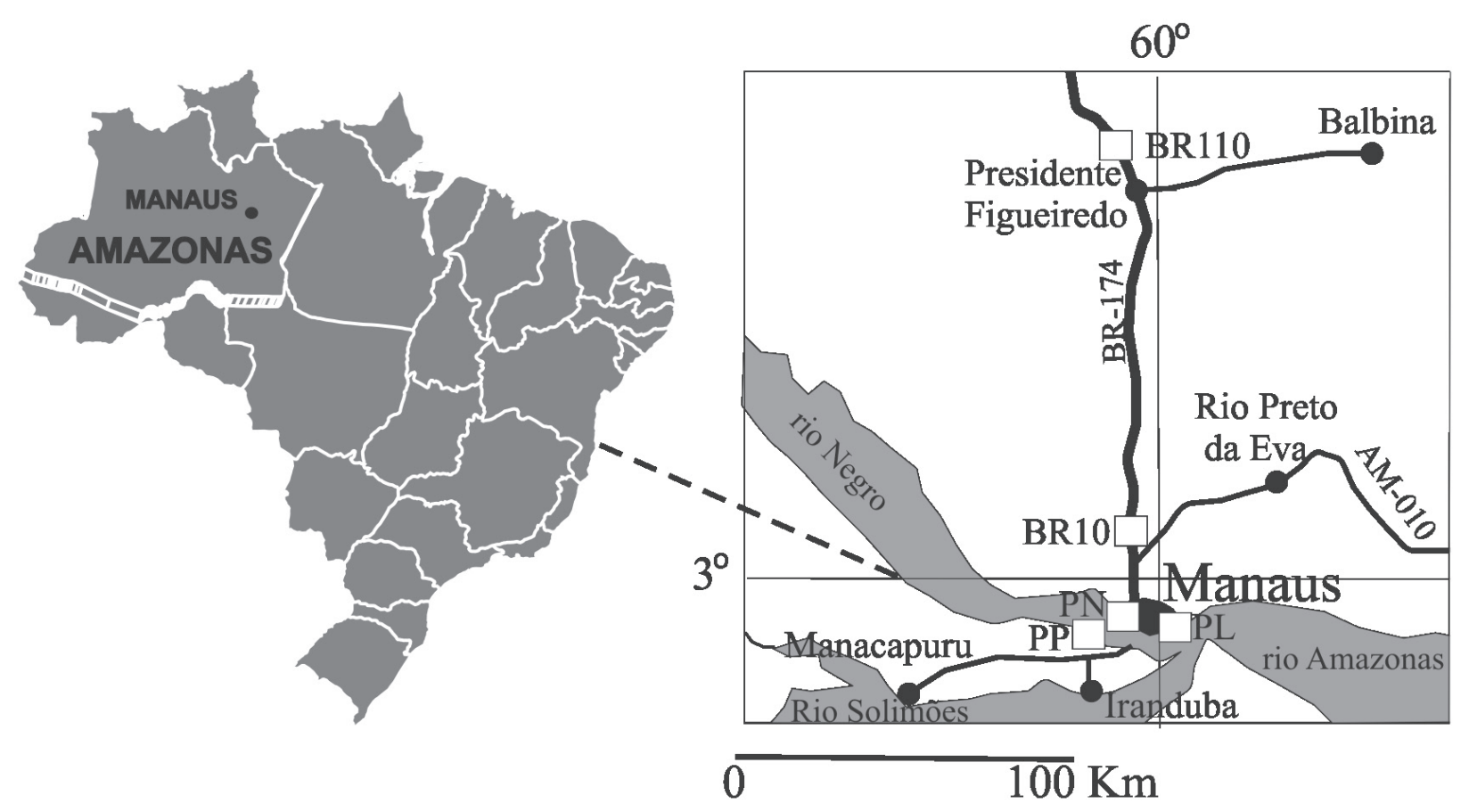

Figura 1- Mapa de localização dos perfis estudados. BR110 - BR174 km 110, BR10 - BR 174 km 10, PN - Perfil da Ponta Negra, PP - Perfil da Ponta das Pedras e PL - Perfil da Ponta das Lajes.

LITOTIPOS E PALEOAMBIENTE As camadas vermelhas dentro da Formação Alter do Chão consistem de arenitos e pelitos friáveis ou silicificados com espessuras que variam entre 0,2 m e $5 \mathrm{~m}$, são contínuas lateralmente por dezenas de metros (Fig. 2 e $3 \mathrm{~A}$ e B). Dois conjuntos foram separados de acordo com a topografia por refletirem distinto posicionamento estratigráfico dentro da unidade. Os perfis situados em cotas topográficas mais baixas $(60 \mathrm{~m})$ e próximos à margem do rio Negro $(\mathrm{PN}, \mathrm{PP}, \mathrm{PL})$ pertencem à base da Formação Alter do Chão, enquanto aqueles da rodovia BR 174 (cotas de até $120 \mathrm{~m}$ ) ao topo desta.

Nestas camadas, além da coloração vermelha intensa, feições de mosqueamento e desferrrificação marcadas por manchas esbranquiçadas são freqüentes (Fig. 2 e 3A e B). Tubos centimétricos com diâmetros de até $1 \mathrm{~cm}$, lisos, meniscados, endicniais e epcniais caracterizam as bioturbações feitas por animais (anelídeos e insetos), enquanto traços verticais com até $3 \mathrm{~cm}$ de diâmetro com formas radiculares, geralmente silicificados e/ou ferruginizados, indicam marcas de raízes (Fig 3 C, D, e E). Os níveis silicificados da PN exibem fósseis de madeira e são comuns aos perfis da base aflorante da Formação Alter do Chão.

As camadas vermelhas estudadas da Formação Alter do Chão foram depositadas em ambientes de planície de inundação, lacustre e algumas delas representam silcretes (Nogueira et al. 1999, Nogueira et al. 2003). As camadas adjacentes aos níveis vermelhos bioturbados são em geral, arenitos esbranquiçados de granulação média a muito grossa, mal selecionados, com grãos subangulosos a angulosos, marcados por acamamento maciço, estratificações plano-paralelas e cruzada-acanaladas, interpretados como depósitos de canais fluviais.

MINERALOGIA Quartzo e caulinita são os minerais principais, seguidos em menor proporção de hematita e goethita. Ocorrem ainda feldspato potássico, principalmente na PL. Neste último, a presença da reflexão em $8,70^{\circ} 2 \theta$ de baixa intensidade indica, ainda, a presença de muscovita e illita. A reflexão $6,7^{\circ}$ $2 \theta$ identifica também esmectita, o que é reforçado pelas análises do MEV-EDS que determinaram a presença de $\mathrm{Na}, \mathrm{Mg}, \mathrm{K}$ e $\mathrm{Fe}$ em estruturas em forma de repolho típica desse mineral (Fig. 3F, G e H). Rutilo e zircão ocorrem como minerais resistatos. A presença de feldspato potássico, muscovita, illita e esmectita indica que esses perfis ainda mantêm suas características primárias preservadas, pois durante a lateritização, que predomina na região, somente caulinita, quartzo e óxi-hidróxidos de ferro são estáveis.

A caulinita predomina nos perfis estudados exceto no da PN onde o quartzo é o mineral mais abundante. Geralmente a caulinita ocorre em cristais isolados, mas também forma estruturas em acordeom (Fig.3 G, H e I). Essa morfologia sugere que pelo menos parte da caulinita deve ter se formado in situ, assim como os cristais em forma de repolho da esmectita, em conseqüência, provavelmente, do intemperismo pos-deposicional dos sedimentos. A hematita está disseminada na rocha e lhe dá a cor avermelhada, enquanto a goethita está restrita aos tubos e é responsável, junto com a hematita, pela preservação das marcas de raízes.

\section{COMPOSIÇÃO QUÍMICA}

Óxidos maiores Os resultados das análises químicas mostram que $\mathrm{SiO}_{2}$ e $\mathrm{Al}_{2} \mathrm{O}_{3}$, seguidos em menor proporção pelo $\mathrm{Fe}_{2} \mathrm{O}_{3}$, são os constituintes mais abundantes (Fig. 4). $\mathrm{O} \mathrm{SiO}_{2}$ destaca-se no perfil da $\mathrm{PN}$ pelos teores em torno de $88 \%$ e tem conseqüentemente os mais baixos de $\mathrm{Al}_{2} \mathrm{O}_{3}$, enquanto nos demais a variação é menos acentuada com tendência da razão entre eles ser em média menor que 4 nos perfis da rodovia BR 174 e PP. Os conteúdos de ferro são inferiores a $12 \%$ e atingem, nas porções esbranquiçadas, menos de $3 \%$. Os mais elevados ocorrem na rodovia BR $174 \mathrm{~km} 110$ e na PP, os quais contêm até 11,9\% e 9,7\%, respectivamente (Fig. 4). Dentre os álcalis destaca-se o $\mathrm{K}_{2} \mathrm{O}$ no perfil da PL com conteúdos entre $3,05 \%$ e $3,81 \%$ e o $\mathrm{Na}_{2} \mathrm{O}$ entre $0,10 \%$ e $0,22 \%$, contudo teores elevados desses ele- 


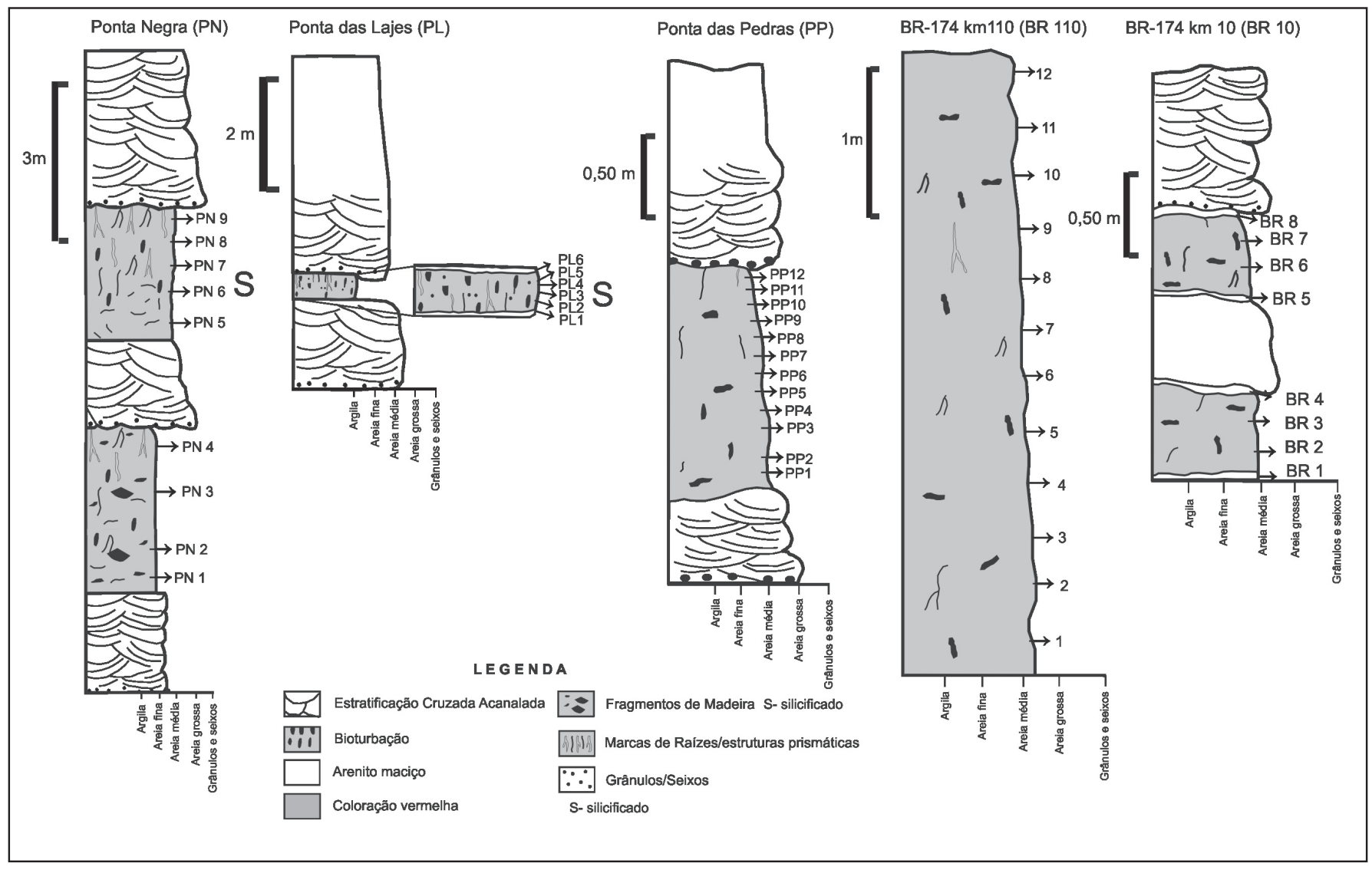

Figura 2: Estruturação dos perfis com arenitos bioturbados. A intesidade da bioturbação é demonstrada pela frequência da simbologia.

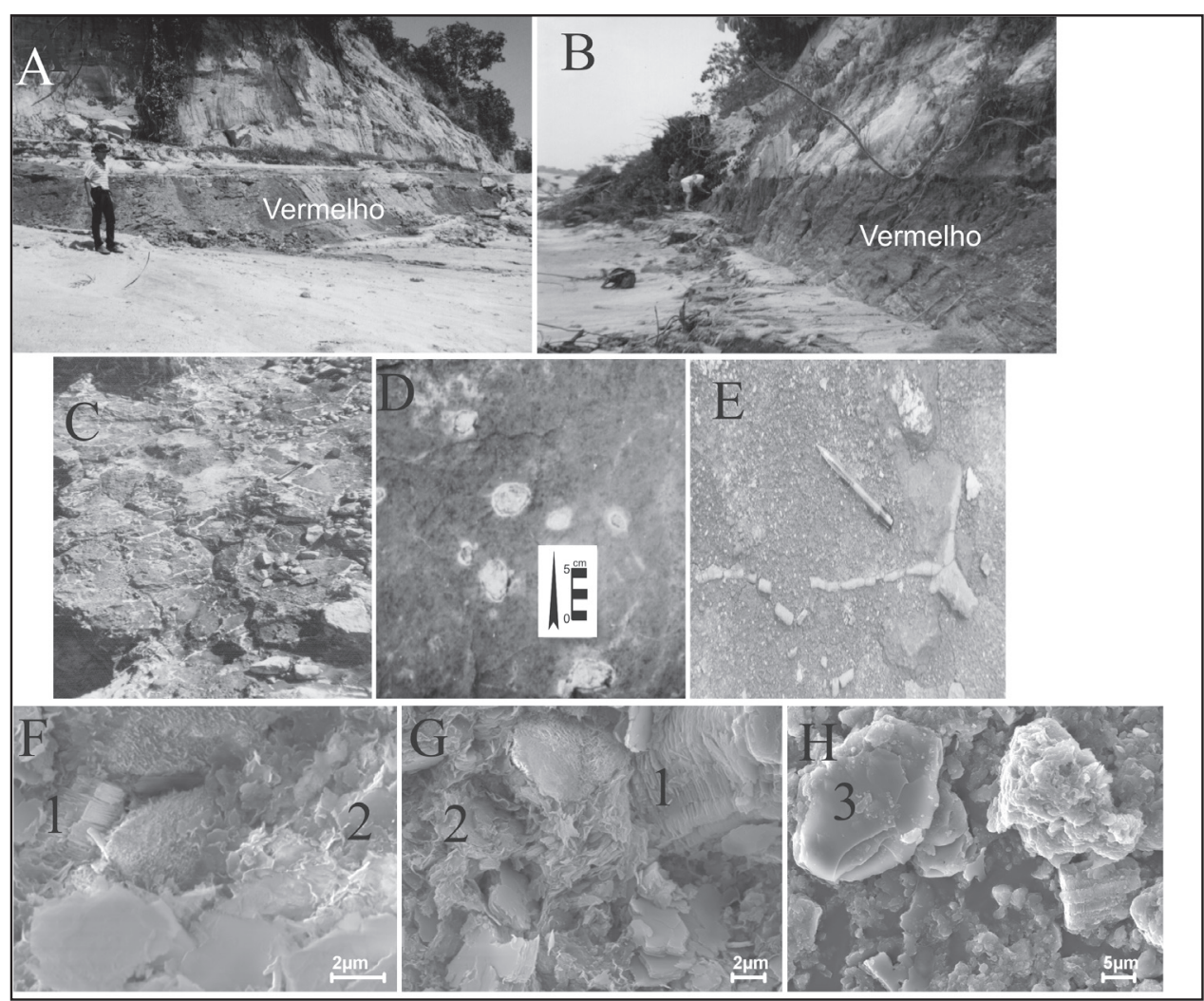

Figura 3: A - Perfil da Ponta das Lajes - PL e B - da Ponta das Pedras - PP ambos com destaque para o arenito vermelho, C e D -marcas de raizes e animais do perfil da Ponta Negra - PN, E - relictos de raízes preservados por cimento de hematita e goethita do perfil da rodovia BR $174 \mathrm{~km} 110$ - BR 110, F, G e H - fotografias obtidas por MEV do perfil da Ponta das Lajes: 1 - estrutura em acordeom de caulinita, 2- esmectita e 3 - muscovita. 


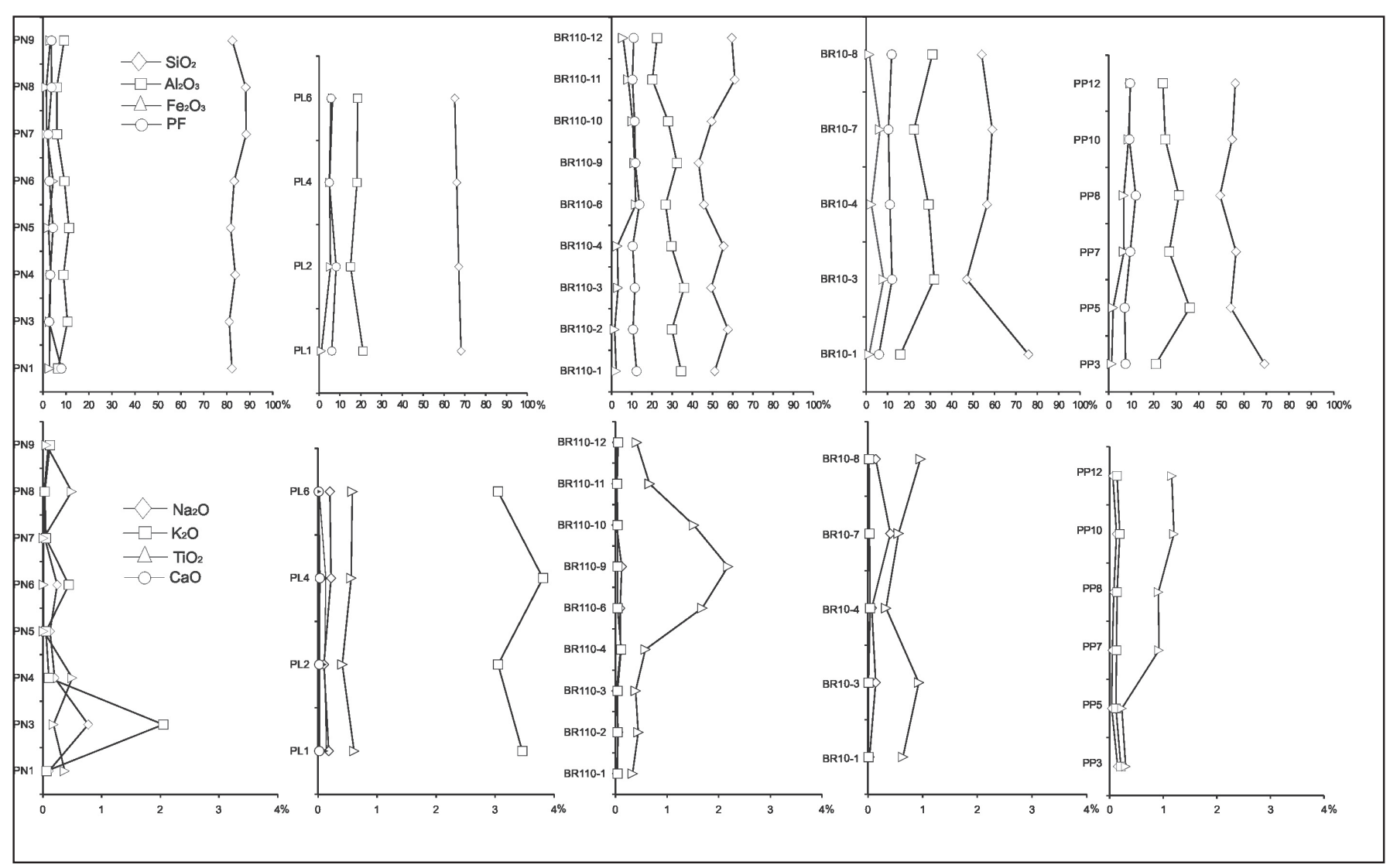

Figura 4: Composição química das camadas vermelhas dos perfis estudados. PN - Ponta Negra, PL - Ponta das Lajes, BR-110 - BR 174/km 110, BR-10 - BR 174/km 10 e PP - Ponta das Pedras.

mentos, mas isolados, ocorrem na base do perfil da PN $(2,05 \%$ e $0,77 \%$, respectivamente). Esses conteúdos mais elevados coincidem com a presença de illita, muscovita, esmectita e feldspato potássico. Nos demais perfis não ultrapassam individualmente $0,44 \%$. CaO e $\mathrm{MgO}$ estão abaixo do limite de detecção (0,01\%). $\mathrm{O} \mathrm{TiO}_{2}$ ocorre entre $<0,01$ e $2,18 \%$ e a perda ao fogo alcança no máximo $13,8 \%$ e reflete, basicamente, a água estrutural dos argilominerais e da muscovita.

A composição química dos perfis estudados não indica estruturação em horizontes, mas as variações acentuadas de teores, especialmente de $\mathrm{SiO}_{2}$ e $\mathrm{Fe}_{2} \mathrm{O}_{3}$ no perfil da rodovia $\mathrm{BR} 174 \mathrm{~km}$ 10 , de $\mathrm{Fe}_{2} \mathrm{O}_{3}$ e $\mathrm{TiO}_{2}$ no da rodovia $\mathrm{BR} 174 \mathrm{~km} 110$ e na PP e de $\mathrm{Na}_{2} \mathrm{O}$ e $\mathrm{K}_{2}^{2} \mathrm{O}$ no da $\mathrm{PN}$ indicam heterogeneidade, provavelmente, relacionadas ao sedimento original.

Elementos-traço No geral os elementos-traço apresentam baixas concentrações, $\mathrm{Ba}, \mathrm{Cr}, \mathrm{Cu}, \mathrm{Ni}, \mathrm{Sr}, \mathrm{V}, \mathrm{Y}, \mathrm{Zn}$ e $\mathrm{Zr}$ estão abaixo da média crustal, enquanto que $\mathrm{As}, \mathrm{Cd}, \mathrm{Pb}$ e Mo são, em parte, superiores a esta. O Sb, Ag, Be, Bi, Li, Sn e W estão em concentrações abaixo do limite de detecção.

$\mathrm{Na}$ maioria dos perfis $\mathrm{V}$ e $\mathrm{Cr}$ são os elementos mais abundantes exceto na PL onde predominam Ba e Y, além de Zn, Sr e Ni (Fig. 5). Esse perfil apresenta o maior conteúdo de elementos-traço, enquanto o da PN o menor. Nos perfis com dois níveis de camadas vermelhas há variações acentuadas na concentração dos elementos-traço, especialmente no da rodovia BR $174 \mathrm{Km}$ 10 com conteúdo maior no nível superior, especialmente para B, $\mathrm{Cr}$, e V. No da PN as variações são menos acentuadas e somente o V é maior no nível inferior em relação ao superior. Nos demais também há variações, $\mathrm{B}, \mathrm{Cr}, \mathrm{Cu}, \mathrm{Hg}, \mathrm{Ni}, \mathrm{Pb}$ e $\mathrm{Zn}$ são bem mais concentrados no topo do perfil da rodovia BR $174 \mathrm{~km} \mathrm{110,} \mathrm{B,}$ $\mathrm{Cd}, \mathrm{Cr}, \mathrm{Cu}, \mathrm{Sc}, \mathrm{V}, \mathrm{Zn}$ e Zr no da PP e Co, Mo e Pb predominam na porção intermediária deste último. Deve-se destacar que no da PL o conteúdo de elementos-traço é muito menor na base que no restante do perfil, contudo dentre aqueles que o caracterizam quimicamente ( $\mathrm{Ba}, \mathrm{Y}, \mathrm{Zn}, \mathrm{Sr}$ e Ni) somente o $\mathrm{Zn}$ se assemelha em conteúdo aos demais perfis. Outras particularidades na distribuição dos elementos-traço: o As está acima do limite de detecção apenas no perfil da rodovia BR $174 \mathrm{Km} 10$ e no topo do Km 110, Co predomina no perfil da PL e em parte do da PP. $\mathrm{O}$ Sc tem valores baixos com distribuição bastante homogênea e apenas para o topo do perfil da rodovia BR $174 \mathrm{Km} 110$ há clara tendência de aumento de teor (Fig. 5).

Elementos terras raras (ETR) Os teores dos ETR, analisados somente na rodovia BR $174 \mathrm{~km} 10$ e no da PN, são mais baixos no primeiro, especialmente nos ETRL. A normalização desses elementos em relação aos condritos resultou em curvas subparalelas com concavidade para cima mais acentuada no da rodovia BR $174 \mathrm{~km} \mathrm{10.} \mathrm{Ambos} \mathrm{têm} \mathrm{suave} \mathrm{anomalia} \mathrm{negativa} \mathrm{em}$ $\mathrm{Eu}\left(\mathrm{Eu} / \mathrm{Eu}^{*} \sim 0,60\right)$, exceto em uma amostra da $\mathrm{PN}$, cuja razão 0,95 caracteriza ausência de fracionamento desse elemento, e ausência de anomalia de Ce (Fig. 6). O fracionamento dos ETRL em relação aos ETRP e entre os ETRP é mais acentuado no perfil da $\mathrm{PN}\left(\mathrm{La}_{\mathrm{n}} / \mathrm{Yb}_{\mathrm{n}}=16,91\right.$ a 28,27 e $\mathrm{Gd}_{\mathrm{n}} / \mathrm{Yb}_{\mathrm{n}}=0,97$ a 3,10), enquanto entre os ETRL é maior na rodovia BR $174 \mathrm{~km} 10\left(\mathrm{La}_{\mathrm{n}} /\right.$ $\mathrm{Sm}_{\mathrm{n}}=7,40$ a 10,78$)$.

\section{INTERPRETAÇÃO DA COMPOSIÇÃO QUÍMICA COM} BASE EM ANÁLISE ESTATÍSTICA A lateritização cenozóica pode ter afetado a geoquímica dos depósitos estudados, contudo a preservação de fósseis de madeira silicificados e as texturas e estruturas sugerem que as modificações mineralógicas e químicas não devem ter sido muito alteradas. Desta forma, a 


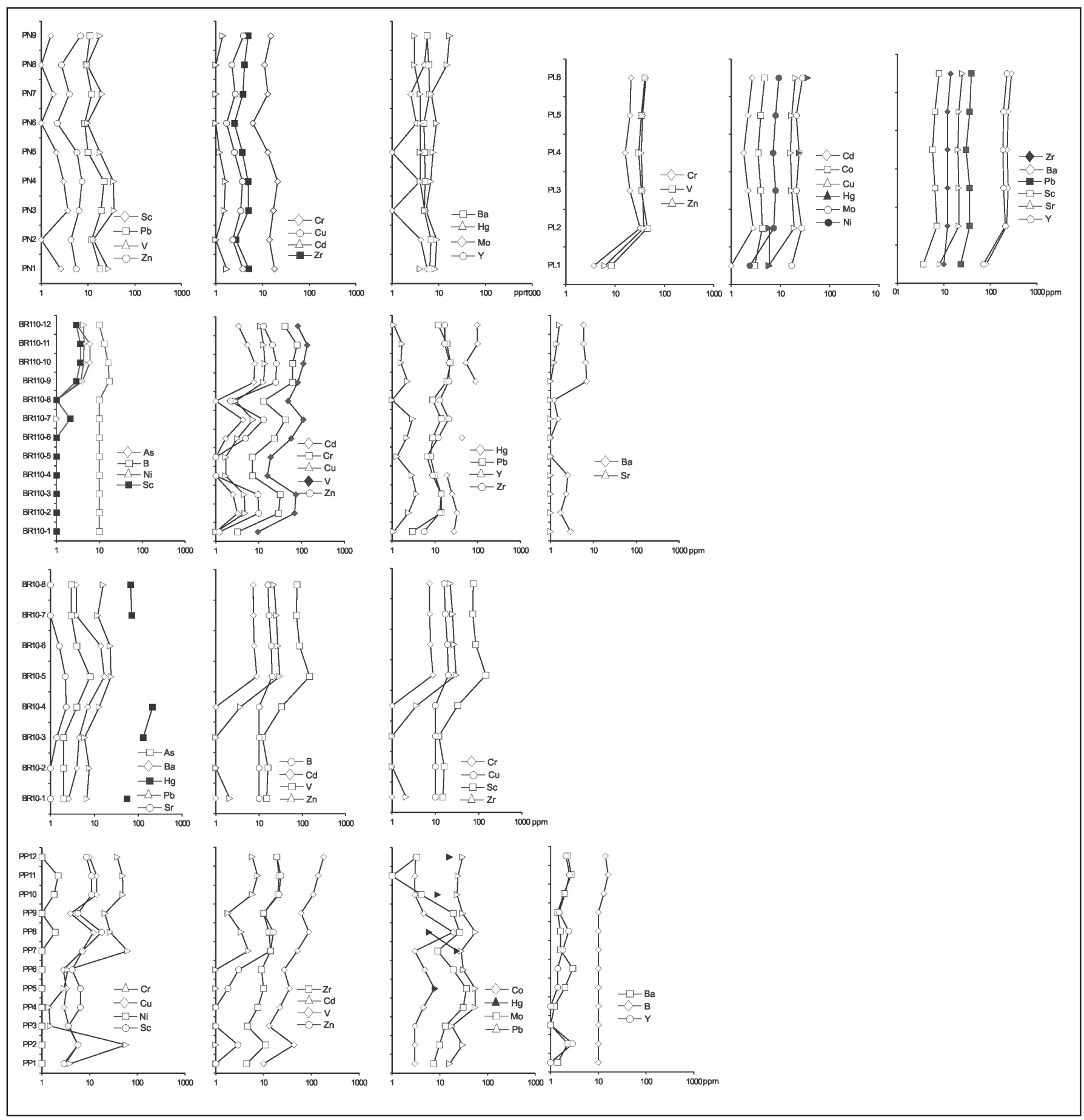

Figura 5: Concentração dos elementos-traços nas camadas vermelhas dos perfis estudados. PN - Ponta Negra, PL - Ponta das Lajes, BR-110 - BR 174/km 110, BR-10 - BR 174/km 10 e PP - Ponta das Pedras.

caracterização geoquímica dos perfis é válida e, assim seus resultados foram integrados via análise estatística por componente principal. O objetivo do uso dessa técnica foi auxiliar na identificação dos elementos que melhor discriminam os perfis estudados e determinar o grau de similaridade entre e ao longo de cada um, gerando uma assinatura geoquímica que pudesse guiar futuros trabalhos com este enfoque. Assim, foram identificados dois fatores que somam $62 \%$ do total da variança, com as seguintes variáveis-elementos mais significativas: no fator $1 \mathrm{Fe}_{2} \mathrm{O}_{3}, \mathrm{TiO}_{2}, \mathrm{Cd}$, $\mathrm{Cr}, \mathrm{V}, \mathrm{Zn}$ e $\mathrm{Zr}$ com carga positiva maior que 0,7 e $\mathrm{SiO}_{2}$ com carga $-0,7$ e no fator 2: $\mathrm{K}_{2} \mathrm{O}, \mathrm{Ba}, \mathrm{Ni}, \mathrm{Mo}, \mathrm{Sr}$ e $\mathrm{Y}$ com carga positiva maior que 0,7 (Tab. 1). Essas cargas elevadas são conseqüências desses elementos serem os que apresentaram maior número de correlações significativas dentre todos os analisados e, portanto os mais relevantes na definição das assinaturas geoquímicas dos perfis. Com base nesses elementos foram obtidas as cargas das amostras que ao serem plotadas em um diagrama xy (Fig.7) permitiram a visualização de três grupos de camadas vermelhas bioturbadas na Formação Alter do Chão: 1- PL, 2- PN e 3- os das rodovias BR $174 \mathrm{~km} 10$ e km 110 e PP. O perfil da PL, com três amostras com cargas positivas nos dois fatores e uma amostra com carga negativa no fator 1 , está localizado no primeiro e segundo quadrante, respectivamente. A posição dessas amostras, com maior variação nas cargas do fator 2 indicam que $\mathrm{K}_{2} \mathrm{O}-\mathrm{Ba}$ - Mo - Ni - Sr - Y é a associação que o caracteriza quimicamente, contudo a variação de cargas entre - 0,6 e 1,5 no fator 1 mos- 


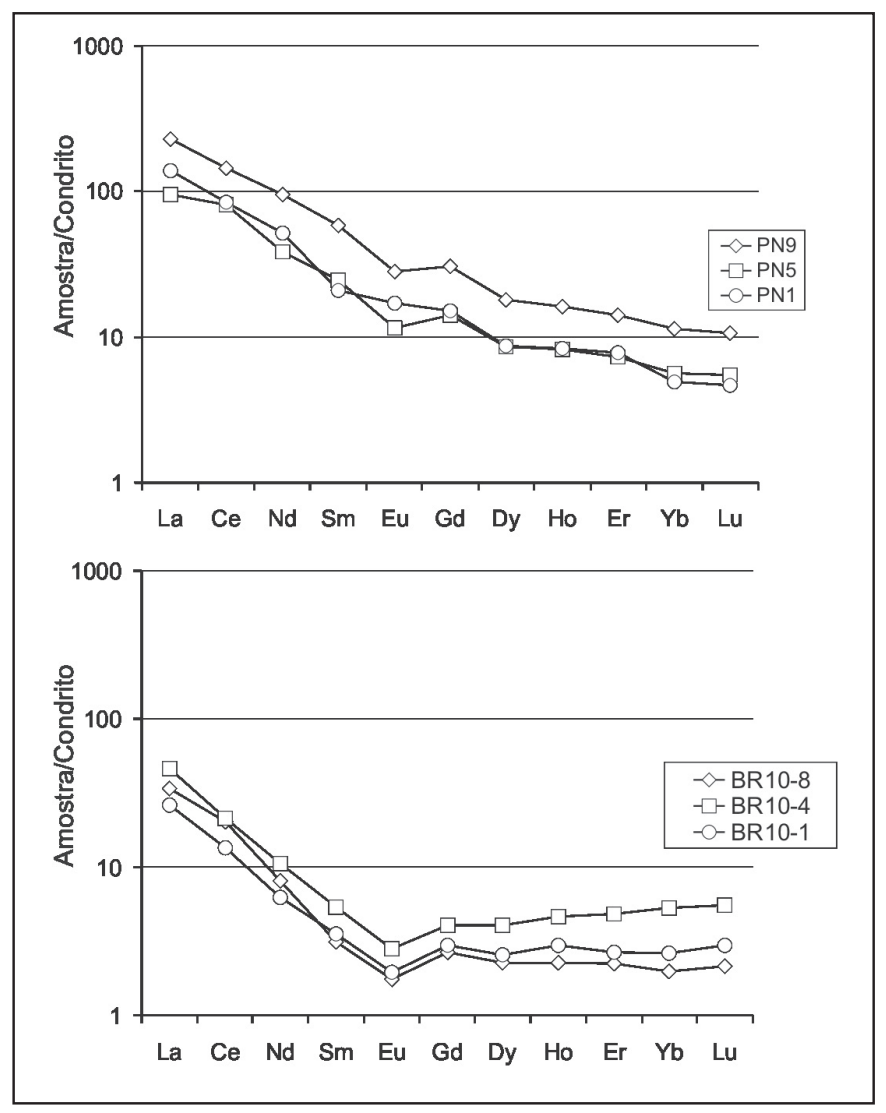

Figura 6 - Curvas dos ETR normalizados segundo os condritos. PN - Perfil da Ponta Negra e BR 10 - Perfil da BR 174/km 10.

tra que a associação $\mathrm{Fe}_{2} \mathrm{O}_{3}-\mathrm{TiO}_{2}-\mathrm{Cd}-\mathrm{Cr}-\mathrm{V}-\mathrm{Zn}-\mathrm{Zr} / \mathrm{SiO}_{2}$ também exerce forte influência em sua composição. As cargas crescentes dos fatores 1 e 2 nesse perfil coincidem com a base e o topo dos perfil, ou seja, conteúdos crescentes de elementostraço e $\mathrm{Fe}_{2} \mathrm{O}_{3}$. A disposição dos demais perfis ao longo do fator 1 demonstram que é a associação $\mathrm{Fe}_{2} \mathrm{O}_{3}-\mathrm{TiO}_{2}-\mathrm{Cd}-\mathrm{Cr}-\mathrm{V}-\mathrm{Zn}$ - $\mathrm{Zr}$, em oposição ao $\mathrm{SiO}_{2}$, que exerce maior influência em sua discriminação. Como o aumento da carga dos fatores das amostras está diretamente relacionado a carga dos elementos positivos e inversamente a dos negativos, o perfil da PN, com os menores conteúdos de elementos-traço mas mais elevados em $\mathrm{SiO}_{2}$, é o que tem as cargas mais negativas no fator 1 . Os perfis da rodovia BR $174 \mathrm{Km} 10$ e Km 110 e o da PP dispostos ao longo do fator 1 , mas sem formar grupos isolados, caracteriza grande espectro de variação química entre eles (Fig. 7).

A geoquímica mostrou que há pelo menos três tipos de depósitos vermelhos bioturbados na Formação Alter do Chão: o perfil da $\mathrm{PN}$ com elevados teores em $\mathrm{SiO}_{2}$, mas baixos em elementos-traço, o da PL com mais $\mathrm{K}_{2} \mathrm{O}, \mathrm{Ba}, \mathrm{Mo}, \mathrm{Ni}, \mathrm{Sr}$ e $\mathrm{Y}$, e os da rodovia BR $174 \mathrm{Km}$ 10, Km 110 e PP com ampla variação química entre eles, mas intermediária entre os dois extremos representados pelos da PN e PL. Essa diferença nas assinaturas geoquímicas reforça a possibilidade de fontes distintas, além dos fatores pedogenéticos pós-deposicionais. Podem ter havido também ação da diagênese e até mesmo do intemperismo mais recente, contudo a influencia desses fatores não foi detectada.

CONSIDERAÇÕES GENÉTICAS E PALEOCLIMÁTICAS Vários fatores como composição da rocha fonte, intensidade do intemperismo, taxa de deposição, seleção granulométrica durante o transporte e deposição, pedogênese pós-deposicional e, finalmente, a diagênese influenciam a composição

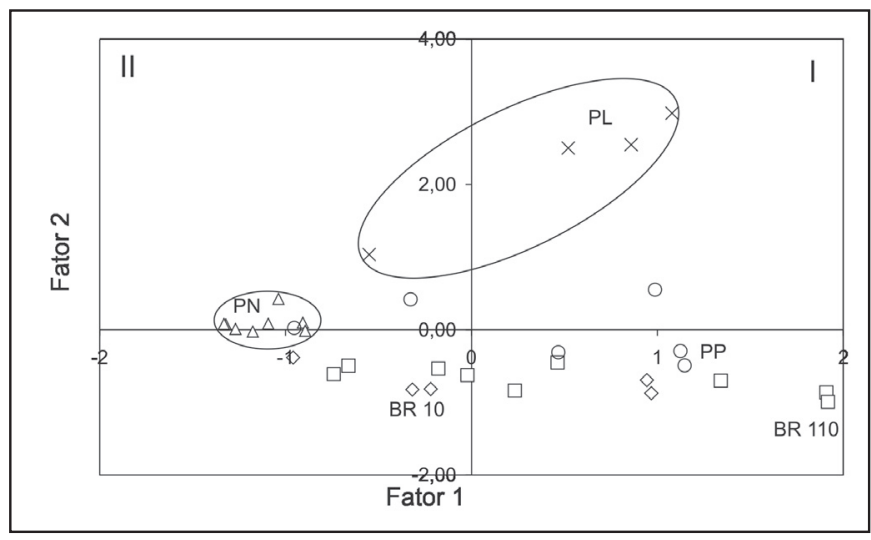

Figura 7 - Análise fatorial das camadas vermelhas dos perfis estudados (PN: Ponta Negra, PP: Ponta das Pedras, PL: Ponta das Lajes; BR 10: rodovia BR $174 \mathrm{Km}$ 10; BR 110: rodovia BR $174 \mathrm{Km} \mathrm{110;} \mathrm{Fator} \mathrm{1:} \mathrm{Fe}_{2} \mathrm{O}_{3}-\mathrm{TiO}_{2}-\mathrm{Cd}-\mathrm{Cr}-\mathrm{V}-\mathrm{Zn}-\mathrm{Zr} / \mathrm{SiO}$; Fator 2: $\mathrm{K}_{2} \mathrm{O}-\mathrm{Ba}-\mathrm{Mo}-\mathrm{Ni}-\mathrm{Sr}-\mathrm{Y}$; I - lo. quadrante, $\mathrm{II}-2 \mathrm{o}$. quadrante)

Tabela 1. Cargas das variáveis obtidas pelo método de extração de componentes principais sem rotação varimax.

\begin{tabular}{|c|c|c|}
\hline Variáveis & Fator 1 & Fator 2 \\
\hline $\mathrm{SiO}_{2}$ & $-0,70$ & 0,32 \\
\hline $\mathrm{Al}_{2} \mathrm{O}_{3}$ & 0,51 & $-0,35$ \\
\hline $\mathrm{Fe}_{2} \mathrm{O}_{3}$ & 0,74 & $-0,15$ \\
\hline $\mathrm{K}_{2} \mathrm{O}$ & 0,09 & 0,87 \\
\hline $\mathrm{Na}$ & $-0,12$ & 0,22 \\
\hline $\mathrm{TiO}_{2}$ & 0,72 & $-0,30$ \\
\hline $\mathrm{PF}^{2}$ & 0,60 & $-0,48$ \\
\hline As & 0,56 & $-0,41$ \\
\hline B & 0,69 & $-0,38$ \\
\hline $\mathrm{Ba}$ & 0,25 & 0,90 \\
\hline $\mathrm{Cd}$ & 0,85 & $-0,29$ \\
\hline Co & 0,20 & 0,23 \\
\hline $\mathrm{Cr}$ & 0,80 & $-0,27$ \\
\hline $\mathrm{Cu}$ & 0,83 & 0,49 \\
\hline $\mathrm{Hg}$ & 0,32 & $-0,39$ \\
\hline $\mathrm{Ni}$ & 0,54 & 0,71 \\
\hline Mo & 0,14 & 0,79 \\
\hline $\mathrm{Pb}$ & 0,42 & 0,54 \\
\hline $\mathrm{Sc}$ & 0,48 & 0,40 \\
\hline $\mathrm{Sr}$ & 0,26 & 0,90 \\
\hline V & 0,78 & $-0,27$ \\
\hline $\mathrm{Y}$ & 0,22 & 0,92 \\
\hline $\mathrm{Zn}$ & 0,79 & 0,51 \\
\hline $\mathrm{Zr}$ & 0,92 & $-0,19$ \\
\hline Variança & 0,34 & 0,28 \\
\hline
\end{tabular}

química e, conseqüentemente a mineralogia de sedimentos clásticos. Estes fatores explicam a diversificação de tipos litológicos em uma mesma unidade geológica como a Formação Alter do Chão. A variação na composição dos arenitos e pelitos vermelhos estudados está basicamente relacionada a presença do cimento silicoso (PN), de feldspato potássico, muscovita, illita e esmectita (PL) que retém $\mathrm{Ba}, \mathrm{Y}, \mathrm{Zn}, \mathrm{Sr}$, e a características intermediárias entre esses dois extermos (BR 174 Km 10, Km 110 e Ponta das Pedras).

O cimento silicoso do perfil da Ponta Negra está relacionado à silcretização pós-deposição (Nogueira et al. 2003), o que su- 
gere rocha hospedeira com boa porosidade e baixa quantidade de material argiloso. Esta maturidade elevada foi conseqüência do ambiente de deposição de alta energia e/ou de intenso intemperismo na área fonte, que propiciou o acúmulo de material detrítico grosso com hematita e ilmenita, enquanto que ambientes de baixa energia favoreceriam a deposição de argilominerais. Esta interpretação vem de encontro ao modelo paleoambiental de planícies abandonadas e rios entrelaçados inferidos para a Formação Alter do Chão (Viera \& Nogueira 1998, Nogueira et al. 1999). A autigênese da caulinita e esmectita que ocorreu durante a pedogênese pós-deposicional de minerais detríticos, liberou sílica e pode ter contribuído para o processo de silicificação comumente encontrado na base aflorante da Formação Alter do Chão. A silicretização e a presença de argilominerais 2:1 indicam que as condições climáticas que prevaleceram durante a deposição e pedogênese foram secas com tendência a úmido (Daemon \& Contreiras 1971, Daemon, 1975 e Rossetti, 2001). Essas condições favoreceram a silicificação e bissialitização com a preservação parcial da muscovita e do feldspato potássico, além da formação, a partir deles de esmectita, illita e caulinita. A silicificação substituiu parcialmente os silicatos primários e os argilominerais e aumentou a proporção de silício no perfil que precipitou como cimento autigênico. A hematita foi incorporada no cimento durante a precipitação e concedeu a coloração vermelha intensa aos depósitos estudados. Nota-se, portanto que os arenitos vermelhos da Formação Alter do Chão são bastante heterogêneos com variações que podem ser atribuídas a fonte dos sedimentos, aos processos pré e pós-deposicionais e as condições paleoclimáticas.

CONCLUSÕES As camadas de arenitos e pelitos vermelhos bioturbados da Formação Alter do Chão, região NE do Estado do Amazonas apresentam influência de duas associações $\mathrm{K}_{2} \mathrm{O}$ - $\mathrm{Ba}$ - $\mathrm{Mo}$ - $\mathrm{Ni}$ - $\mathrm{Sr}-\mathrm{Y}$ e $\mathrm{Fe}_{2} \mathrm{O}_{3}-\mathrm{TiO}_{2}-\mathrm{Cd}-\mathrm{Cr}-\mathrm{V}-\mathrm{Zn}-\mathrm{Zr} / \mathrm{SiO}_{2}$ que combinadas refletem três assinaturas geoquímicas: 1- perfil com elevados teores em $\mathrm{SiO}_{2}$ e baixos em elementos-traço (PN); 2- maior conteúdo de $\mathrm{K}_{2} \mathrm{O}, \mathrm{Ba}, \mathrm{Mo}, \mathrm{Ni}, \mathrm{Sr}$ e Y (PL); e 3- com ampla variação química, mas intermediária entre as assinaturas 1 e 2 (BR 174 Km 10, Km 110 e PP). Essa diferenciação é atribuída as variações da rocha fonte, seleção granulométrica durante o transporte e deposição e pedogênese pós-deposicional. Estas variações ocorreram em paleambiente de planícies abandonadas e rios entrelaçados sob condições de clima seco com tendência a úmido durante o Cretáceo na Bacia do Amazonas.

Agradecimentos Ao CNPq projetos no. 520243/98-6 e 550306/01-3, a CAPES pela concessão de bolsa para o segundo autor, ao curso de pós-graduação em Geociências da UFAM pelo apoio logístico e ao Prof. Dr. Werner Truckenbrodt pela leitura crítica do texto.

\section{Referências}

Albuquerque O.R. 1922. Reconhecimentos geológicos no Valle do Amazonas. In: SGMB, 3, Rio de Janeiro, Boletim, 84p.

Braun J. J., Pagel M., Muller J. P., Bilong A. M., Guillet, B. 1990. Cerium anomalies in lateritic profiles. Geochemistry et Comochemistry Acta, 54:781-795.

Caputo M.V., Rodrigues R., Vasconcelos D.N.N. 1972. Nomenclatura estratigráfica da Bacia do Rio Amazonas. In: SBG, Cong. Bras. Geol., 26, Belém, Anais, v.3, p.35-46.

Cunha P.R.C., Gonzaga F.G., Coutinho L.F.C., Feijó F.J. 1994. Bacia do Amazonas. Boletim de Geociências de Petrobrás, 8:47-55.

Daemon R.F. 1975. Contribuição à datação da Formação Alter do Chão, Bacia do Amazonas. Rev. Bras. Geociências, 5:58-84.

Daemon R.F., Contreiras C.J.A. 1971. Zoneamento palinológico da Bacia do Amazonas. In: SBG, Cong. Bras. Geol., 25, São Paulo, Anais, v.3, p.79-88

Derby O.A. 1879. Contribuições para a geologia da região do baixo Amazonas. Archivos do Museu Nacional, 3:77-104.

Dino R, Silva O.B., Abrahão D. 1999. Caracterização palinológica e estratigráfica de níveis Cretáceos da Formação Alter do Chão, Bacia do Amazonas. In: Simp. sobre o Cretáceo do Brasil, 5, Rio Claro, Boletim de resumos expandidos, p. 557-565.

Kistler P. 1954. Historical resume of the Amazon Basin. PETROBRAS/ RENOR, Belém, Rel. Interno (inédito).

Morey G.B., Setterholm D.R.1997. Rare earth elements in weathering profiles and sediments of Minnesota: implications for provenance studies. Journal of Sedimentary Research, 67:105-115.

Nogueira A.C.R., Vieira L.C., Suguio K. 1999. Paleossolos da Formação Alter do Chão, Cretáceo-Terciário da Bacia do Amazonas, regiões de Presidente Figueiredo e Manaus. In: Simp. sobre o Cretáceo do Brasil, 5, Rio Claro, Boletim de resumos expandidos, p. 261-266.
Nogueira A.C.R., Silva Júnior J.B.C. da, Horbe A.M.C., Soares J.L., Monteiro A.D. 2003. A gênese dos níveis silicificados da Formação Alter do Chão, Cretáceo Superior da bacia do Amazonas. In: SBG/ NO, Simp. Geol. Amaz., 8, Manaus, Boletim de resumos expandidos, CDRom.

Pereira E.R. 1988. Possibilidades metalogenéticas na região do Domo de Monte Alegre - PA. In: SBG, Cong. Bras. Geol., 35, Belém, Anais, v.1, p.286-295.

Rossetti D. F., Truckenbrodt W., Santos Jr. A.E. 2001. Clima do cretáceo no meio-norte brasileiro. In: D.F. Rossetti \& W. Truckenbrodt (eds.) O cretáceo na Bacia de São Luiz-Grajaú. Museu Paraense Emílio Goeldi, 67-76.

Souza M.M. 1974. Perfil geológico da BR-174 (Manaus-Boa Vista) no trecho: Manaus - Serra do Abonari. In: SBG, Cong. Bras. Geol., 28, Porto Alegre, Anais, v.2. p. 75-86.

Souza M.M., Medeiros M.F. 1972. Contribuição ao estudo sedimentológico da região de Manaus. In: SBG, Cong. Bras. Geol., 26, Belém, Anais v.1, p.129-140.

Vieira L.C. \& Nogueira A.C.R. 1998. Petrografia dos arenitos da Formação Alter do Chão, Cretáceo-Terciário da Bacia do Amazonas, Praia da Ponta negra, Manaus. In: ASSER, Cong. Inic. Cientif. , 3, São Carlos, Boletim de resumos, p. 165.

Wanderley Filho. J. R. 1991. Evolução estrutural da Bacia do Amazonas e sua relação com o embasamento. Master's Thesis, Centro de Geociências, Universidade Federal do Pará, Belém, p.125.

Manuscrito A1509 Aprovado em 13 de setembro de 2006 